\title{
ENHANCING THE RT STUDENT CLINICAL EXPERIENCE: NEWCASTLE MATER HOSPITAL RADIATION ONCOLOGY DEPARTMENT
}

\section{Naomi Chapman BApSc(MRS),}

Clinical Consultant Educator Newcastle Mater Misericordiae Hospital, NSW Australia. Conjoint Teaching Fellow, The University of Newcastle, Clinical Associate University of Sydney, Member Australian Institute of Radiography

Address: $\quad$ RT Education, Radiation Oncology Department

Newcastle Mater Misericordiae Hospital

Locked Bag 7

Hunter Region Mail Centre

NSW Australia 2310

Email: $\quad$ mater.rteducation@mater.health.nsw.gov.au

Phone: $\quad 61249211814$

Fax: $\quad 61249211129$

\section{Sharon Oultram RT(T)}

Clinical Consultant Educator Newcastle Mater Misericordiae Hospital, NSW Australia. Conjoint Teaching Fellow, The University of Newcastle, Clinical Associate University of Sydney 


\section{ABSTRACT}

In 2004, the New South Wales (NSW) State Government in Australia provided funding for five clinical consultant, radiation therapist positions, specialising in clinical education. These Radiation Therapy Educator (RTEs) positions where developed in response to the attrition rate in Medical Radiation Science (MRS), Radiation Therapy (RT) courses in NSW and the subsequent impact on qualified radiation therapist numbers. The Radiation Oncology Department at the Newcastle Mater Hospital (NMMH) was granted three year funding for one RTE position.

The RTEs at the NMMH produced a comprehensive program to support undergraduate MRS students, the RTEs utilised concepts regarding clinical education, adult learning and preceptorship in the development of the program. The program involved implementation of an orientation program and department wide preceptors to facilitate learning in the clinical environment. The undergraduate program was adapted specifically to student learning utilising needs assessments, learning contracts and additional specialised tutorials. This assisted the department to bridge the theory practise gaps in the students clinical experience.

Evaluation of the program was focused on establishing baseline data, where little had existed previously and in the long term, substantiating the implementation of the RTE position. Preliminary data indicates that the program, although still in it's infancy, appears to be addressing the needs of the students resulting in enhancement of their clinical experience, whilst also providing a continual process for the program to develop and improve.

\section{INTRODUCTION}

In NSW in 2002 there were 30,448 new cases of cancer producing 2,338 deaths. Of these cases four sites accounted for $59 \%$ of new cancers in males. These were cancers of the prostate $(24 \%)$ and colorectal $(13 \%)$, melanoma of skin $(12 \%)$ and lung $(10 \%)$. Whilst in women, four sites accounted for $58 \%$ of new cancers. The most common were cancers of the breast (29\%) and colorectal (13\%), melanoma of skin (9\%) and lung cancer (7\%). Radiotherapy is playing an increasing role in the management of all these prevalent diseases, creating an increasing demand on the existing radiotherapy services (1) 
During this period however, Radiation Oncology Departments often face staff shortages in conjunction with this increase in demand for services. Baume (2) attempted to examine these staff shortages highlighting student attrition rates as one major contributor to the staffing crisis. Baume identified poor clinical experience early in the undergraduate course to be a contributing factor towards this attrition rate and therefore created a need for the enhancement of the student clinical experience (2).

\section{AIM}

The aim of this paper is to outline the initiatives developed by the Radiation Therapy Educators at the Newcastle Mater Hospital, present evaluations used to assess the performance of the initiatives and provide preliminary data on the feedback obtained to date.

\section{BACKGROUND}

In 2000, the Newcastle Mater Misericordiae Hospital (NMMH) Department of Radiation Oncology commissioned an internal review, which raised key issues regarding Medical Radiation Science (MRS) student training. At the time, the majority of staff felt "that the issue of student training needed attention", the department had "failed to provide a suitable education program for undergraduate radiation therapists" and that students reported that they did "not feel welcome when attending the department for clinical placements" (3, p. 25).

In June 2002 the Vision for Radiotherapy Report chaired by Peter Baume was delivered. This inquiry reviewed the status of the profession on many fronts such as, staffing, training and equipment to name a few. It highlighted that each year in Australia there were approximately 10,000 patients who did not receive radiotherapy, who indeed may have benefited from it. This and the long waiting times for access to RT services were attributed to by the lack of Radiation Therapists (RTs), Radiation Oncologists and Medical Physicists (4).

The shortage of RTs was investigated in depth, highlighting staff attrition rates, student intake and student attrition as some key areas of interest in addressing the critical staff shortage in the current climate. The report highlighted that, there was little problem in attracting students to the undergraduate programs, but there was a problem in student retention once enrolled in the program (4). Difficulty in retaining students was attributed to two primary factors, firstly some students being unaware of what is involved in a career in 
Radiotherapy and the patient sample that they will be required to work with and also poor experiences in a clinical facility. The latter was identified to be a direct result of staff shortages and workload pressures in the clinical departments. The capabilities of departments to provide adaptive and well managed clinical placements for undergraduate students were seen as a limiting factor, primarily due to lack of staff to facilitate the students and their increasing numbers (2).

As a direct result of these findings in 2003, the New South Wales (NSW) government funded Radiation Therapy Educator (RTE) positions in ten departments within the state. These positions were developed to address issues in education, professional development and training over the entire cross-section of staff, from undergraduate students, Professional Development Year staff to qualified and allied health professionals. A large component of the work for the RTE pertains to the undergraduate student. The RTEs at the NMMH have focused the first period of employment specifically on the enhancement of undergraduate student clinical experience.

Many papers and text over the years have addressed the issues of clinical enhancement and preceptorship of various undergraduate students from various health disciplines, primarily nursing. Virtually no literature involves the discipline of radiation therapy, however there tends to be consensus and focus on the following concepts in clinical education.

Raisler et al. (5) identified the following as key characteristics of effective teaching; being well organised, providing clear instructions, informing the students, the centres expectations for an acceptable student and including an orientation to facilities.

All of the above elements can be addressed in a formal orientation to a department. Orientation to learning is a key component of adult learning. The student will focus on immediate issues and have information to problem solve those issues and resolve conflicts with expedience (6). Kaviani and Stillwell (7, p.220) have noted that students "may not be completely aware of their own learning needs and require assistance identifying them". It is during a formal orientation that a needs assessment can be performed to identify the expectations of the learner at the different levels of development. Orientation ensures the learner is informed regarding mode and content to be assessed and information relating to the expectations of the student. The level of competence expected can be communicated, 
which is vital to enabling the student to set realistic goals and objectives for their clinical block (8). During this process the student may also indicate what form of feedback is most helpful for them.

These may include; immediate verses delayed, task specific and verbal rather than written (5).

Teaching in a practical and engaging manner is also identified as an essential component to an effective clinical teaching program (6). Tutorials, used as a form of structured learning experience, can fill the gaps from experiences that may not be encountered or be at a suitable level in clinical practise, but are imperative to the development of the student (5). Workshops and mini or informal education sessions can be very effective and allow a greater practical component to the students learning.

Regular, informal and structured, formal feedback is an essential part of any adult education setting, as a method of helping students achieve their learning objectives. Sachdeva (8) discusses the effectiveness of feedback being accompanied by the establishment of goal setting tasks. The establishment of specific goals from direct feedback steers the learner to concentrating on and achieving desired outcomes. Feedback is not only important for student development but also for program and clinical education development. Continuously reflecting on teaching successes and failures is imperative to maintain an efficient and dynamic program (5).

\section{UNDERGRADUATE STUDENT CLINICAL PROGRAM}

The RTEs at the NMMH have had a blank slate to draw on to initiate a system that would not only enhance the student experience, but also motivate qualified RTs in the area of student education. The undergraduate program consist of the following elements.

\section{Orientation Program}

The Orientation program is designed to be a thorough introduction to the clinical environment. The orientation ensures that valuable clinical time is not used delivering information to the students that can otherwise be given out of the clinical setting. A three-hour orientation session is delivered to all students entering the department. It is tailored to each of the three years of clinical practice blocks. The orientation program contains the following key elements, staff structure and patient pathways, equipment and techniques and 
manual handling summary. Additionally, student duties and expectations, introduction to the undergraduate and preceptor program are discussed. Feedback mechanism are outlined and the orientation is completed with a department tour.

\section{Student Pack}

Prior to the students' arrival at the NMMH, a Student Pack is compiled. This package of resource material is provided to each student at the orientation session. It was developed to ensure the students receive all relevant material, with explanation, at the beginning of their clinical block. It was designed to supplement information provided by the universities. It includes rosters and in-service timetables in addition to the items mentioned below:

Departmental Documentation

This enables the student to become familiar with the format and documentation this department utilises prior to being placed in a clinical situation, as well as providing them with an overview of its practical use in everyday work process.

\section{NMMH Student Handbook}

The handbook contains the Vision and the Mission Statement for the department, the history and future of the department, the referral demographics, departmental profiles, hours of operation and staff facilities.

Needs Assessment Form

Needs Assessments are designed to allow the RTEs the opportunity to respond to the students perceived needs. The Needs Assessment form is mailed or emailed to the prospective students upon receipt of the list of names from the universities. The assessment form addresses three major issues:

1. Learning objectives, report and/or assignment topics the student wishes to focus on during the clinical block.

2. Areas the student is interested in learning about eg. Chemotherapy, brachytherapy, surgery, etc.

3. A reflective comment post-placement to determine if needs were met.

The student roster can be adjusted to accommodate student needs. Specific tutorials can be planned and researched.

Specialist consultants can be scheduled to present information to the students as needs arise.

Weekly Objective Form

This is a form of learning contract, to be completed by the student in consultation with the area preceptor. The majority 
of the form is completed on the first day of each new rotation. At this time, the student will nominate individual learning objectives for the week and specific tasks to be focused on. At the end of the week, the form will be completed, providing feedback regarding the week's rotation. This form implies responsibilities for both the student and preceptor.

Formative Evaluation Form

Both Universities of Sydney and Newcastle utilise student evaluation tools. Sydney University uses a weekly formative evaluation form where no mark values apply. The final summative evaluation produces the student's final clinical mark for the entire block. Newcastle University utilises weekly summative evaluations to which marks do apply. The final clinical block mark is an average of marks from each week. The RTEs use each system as prescribed by the respective universities, however a Formative Supplementary Evaluation form has been constructed to give the preceptor and student a mechanism, if required, to provide mid-week feedback without application of marks. The form is primarily for constructive feedback if the preceptor wishes to highlight where remedial work is required on the student's part. Most students are concerned regarding final grades, if appropriate and timely feedback is provided the student is then given the opportunity to respond to the feedback and implement change in order to improve.

\section{Tutorials}

Tutorials with the RTEs have been introduced as another method to enhance the clinical experience for students. Tutorials are designed to provide step-by-step instruction regarding techniques, the rationale and the opportunity to practice without having the stress of working under actual treatment or planning conditions. The student can then confidently apply their new knowledge and skills to the clinical situation. The RTs working with the student will in turn be more confident in the student's knowledge and abilities and therefore reduce the student's performance anxiety.

\section{Professional Development In-Service Education}

Students are encouraged to attend professional development education sessions as well as technical, quality assurance and research meetings held in the department. By attending these sessions, the students are exposed to information, studies and trials that will enhance their learning. Students also can observe the multi- 
disciplinary approach that is applied to cancer treatment as all meetings involve medical, nursing, scientific and allied health care professionals.

\section{Evaluation of the Student Clinical Program}

As previously mentioned, the evaluation of the initiatives developed by the RTEs and the department's effectiveness in enhancing the clinical experience for undergraduate students has begun, using evaluation forms and a debrief focus group. One such form evaluates the placement as a whole, whilst another examines the effectiveness and performance/impact of the preceptor program on the clinical experience of students attending the NMMH. Data collection not only guides the program towards improvement, but it provides data to substantiate the need for dedicated RTEs. There is little quantitative data to date and the RTEs hope to at least produce departmental baseline data to begin its study of effectiveness.

\section{RESULTS}

\section{Orientation Program Feedback}

All students that undertake the department orientation are required to fill in a feedback form. Participants were asked to rate different elements of the orientation on a line scale from zero (not at all) to ten (very). The mean of the responses were as follows.

Did you find the session of interest?

Did you find the session relevant to you? 9.2 Was the hands on experience useful? $\quad 8.8$

Feedback received from students since the inception of the orientation program has been most favourable. Some student comments include, "feeling less nervous because I knew what was expected of me" in regards to clinical practice and, "knowing what is to be expected from us as students during this clinical was an advantage so we know what we can do and what we shouldn't."

\section{Overall Clinical Evaluation}

This evaluation is for the students to give feedback to the RTEs and the department regarding their experience during the clinical block. This evaluation form delves into a number of areas. Some of those areas are:

- Achievement of set objectives 
- Rostering and duration of time spent in each area

- Access to patients, patient information, equipment and other resourses

- Teamwork

- Pace and style of learning

- Valuable features of the placement

- Suggested improvements

The form states that the information collected may be used for statistical analysis and possibly for publication. It is entirely voluntary, but by completing the form, permission from the student is implied.

Feedback to date reveals that:

- All students reported to have enjoyed their time in the department, were able to achieve the set objectives of their clinical placement and had adequate access to patients, patient information, equipment and other resources.

- $96.3 \%$ found the orientation and weekly meetings worthwhile, no respondents found them a waste of time.

- $96.3 \%$ of students felt the roster met their needs. Most students felt they would like more time in planning.

- $92.6 \%$ of students agreed that they felt like part of the team.

\section{Student Debrief and Discussion Group}

The debrief sessions with students facilitates responses from students otherwise unobtainable via written format. When the narrative from these debriefs is correlated with the results from the written evaluation, often a greater understanding of the issues is displayed

Some student comments have indicated the positive role a structured approach has played in their clinical development:

- "Having the student supervisors and a room to go to talk to them, is relieving when you have a problem"

- "Beneficial to have a port of call and support"

- "It was very organised and it was good to see that someone had planned our whole two weeks for us"

- "Helped by showing me how things worked prior to me using them so I didn't feel as pressured when using them"

- "WOF is a good tool to enhance communication between us and the preceptors" 


\section{CONCLUSION}

Responding to the specific needs highlighted by various investigators, the RTEs at the NMMH have developed a student program. The program recognises and utilises adult education principles and guidelines evident in the literature, as discussed, in order to enhance the student clinical experience within the department. Preliminary feedback has allowed for the establishment of baseline data, which will assist in any future program evaluation. Preliminary feedback has shown encouraging results that the program is beginning to meet the needs of the undergraduate student and enhance their clinical experience. In the future, the RTEs plan to examine programs annually to ensure the programs adapt with the requirements of the respective universities and students.

\section{REFERENCES}

1. NSW Cancer Council Statistics. In: NSW Cancer Council; 2002.

2. Baume P. Radiation Oncology Inquiry. A vision for radiotherapy. Canberra: Commonwealth of Australia; 2002.

3. Simpson M. Radiotherapy Service Review: Newcastle Mater Hospital. Newcastle: Newcastle Mater Hospital; 2000.

4. Barton MB, Peters LJ, Kenny LM. Radiotherapy in Australia one year after the Baume report: vision or mirage? Med J Aust 2004; 180(2):55-6.

5. Raisler J, O'Grady M, Lori J. Clinical teaching and learning in midwifery and women's health. J Midwifery Womens Health 2003;48(6):398-406.

6. Certificate IV in Assessment and Workplace Training. In: Australian National Training Authority; 1998.

7. Kaviani $N$, Stillwell $\mathrm{Y}$. An evaluative study of clinical preceptorship. Nurse Educ Today 2000;20(3):218-26.

8. Sachdeva AK. Use of effective feedback to facilitate adult learning. J Cancer Educ 1996;11(2):106-18. 